\title{
Asymptomatic Presentation in Arrhythmogenic Right Ventricular Cardiomyopathy: a Case Report.
}

\section{Anish Hirachan, Ranjit Sharma, Prabesh Neupane, Milan Gautam, Ram Kumar Ghimire.}

Nepal Mediciti Hospital, Kathmandu, Nepal.

Corresponding Author:

Anish Hirachan

Nepal Mediciti Hospital, Kathmandu, Nepal.

E-mail: hirachananish@gmail.com

ORCID ID NO: 0000-0001-7714-7295

Submitted date: $28^{\text {th }}$ December 2020

Accepted date: $12^{\text {th }}$ April 2021

Cite this article as: Hirachan A, Sharma R, Neupane P, et al. Asymptomatic Presentation in Arrhythmogenic Right Ventricular Cardiomyopathy: a Case Report. Nepalese Heart Journal 2021; Vol 18 (1): 65-66.

\section{Abstract}

\begin{abstract}
Arrythmogenic right ventricular cardiomyopathy (ARVC) is an inherited cardiac condition leading to one of the common cause for sudden cardiac death in young individuals. It is characterized by fibrofatty replacement of the cardiac myocytes predominantly the right ventricle. As the presentation can be varied and very nonspecific, it often leads to delay in diagnosis and specific treatment. Here, we present a 30 year male who was asymptomatic and detected to have electrocardiograph (ECG) and echocardiographic abnormalities suggestive of ARVC. Further, a cardiac imaging with cardiac MRI confirmed the diagnosis of ARVC highlighting the importance of multimodality imaging.
\end{abstract}

Keywords: Arrhythmogenic Right ventricular Cardiomyopathy; Asymptomatic Presentation.

DOI: https://orcid.org/10.3126/njh.v18i1.36791

\section{Case}

A 30 year old male teacher by occupation presented for a routine health examination at our institute. He had no symptoms of breathlessness, chest pain, dizziness or palpitation. His clinical examination was unremarkable with normal vital parameters. There was no significant history of cardiac disease or any sudden cardiac death in the family. He had been active and no any comorbidities till date. His routine electrocardiograph (ECG) showed repolarization abnormalities in form of $\mathrm{T}$ wave in version in leads $\mathrm{V} 1-\mathrm{V} 4$ and lead III (major). (Image 1)

Image 1: ECG showing T wave inversion in V2-V4.

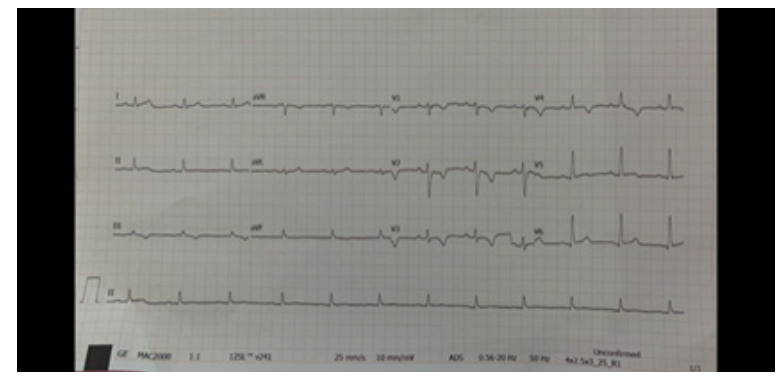

His echocardiogram revealed dilated right atrium (RA) and right ventricle (RV) with dyskinetic RV apex along with dilated right ventricle outflow tract (RVOT):(parasternal long axis (PLAX) dimension-51mm, parasternal short axis dimension (PSAX)-53mm, moderate tricuspid regurgitation with mild pulmonary hypertension Tricuspid annular plane systolic excursion (TAPSE)-19mm, RV systolic tissue doppler velocity (RV s TDI) $9.9 \mathrm{~mm}$ and normal Left Ventricle (LV) dimension and systolic function (major). He further underwent a cardiac MRI (plain and gadolinium enhanced) which showed a markedly dilated right ventricle and markedly hypokinetic free walls of the right ventricle with associated thinning of the RV wall and patchy fatty areas noted in the right ventricular wall. Late gadolinium enhancement scan showed patchy enhancement of the right ventricle free wall. Right ventricle apex and mid free wall showed hypertrabeculated appearance. (Major) These findings with compared to the Task Force criteria (2010) for Arrythmogenic right ventricular cardiomyopathy (ARVC) fulfilled for the definite diagnosis of ARVC with 2 major (both Echo and cardiac MRI/ECG). The index patient was totally asymptomatic and hence was offered no active treatment but with close follow up with monitoring of any new symptoms. However, all first degree relatives of the index case have been advised for a screening echocardiographic examination.

(a) Nepalese Heart Journal. Nepalese Heart Journal retain copyright and works is simultaneously licensed under Creative Commons Attribution License CC - By 4.0 that allows others to share the work with an acknowledge of the work's authorship and initial publication in this journal 
Image 2: Cardiac MRI showing dilated right ventricle with thinned right ventricular wall with features of fatty infiltration and late gadolinium enhancement .
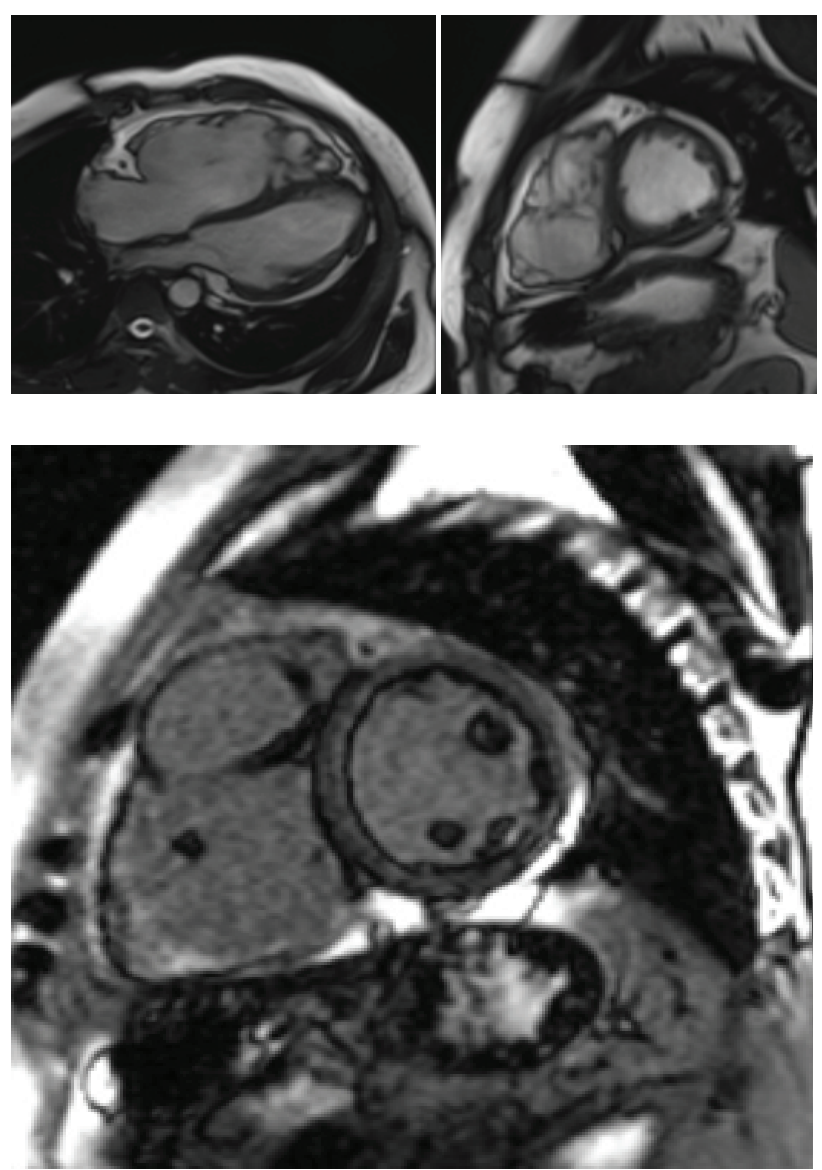

\section{Discussion}

In 1978 Frank and Fontain first described ARVD (arrhythmogenic right ventricular dysplasia) in six patients presenting with ventricular tachycardia (VT). ${ }^{1}$ It was defined as "total or partial replacement of right ventricular muscle with fibro-fatty tissues associated with left bundle branch (LBBB) morphology arrhythmias". True incidence of this disease is not known but the prevalence is approximately 0.02 $0.1 \%$ in the general population.

The diagnosis of ARVC is made in $80 \%$ of cases in patients below the age of 40 years. Men are more commonly affected. Hence, it should be suspected in young patients presenting with unprecedented history of syncope, cardiac arrest or arrhythmias. The natural history of ARVC depends on the rate of progression of RV dysfunction and the clinical presentation. The mortality rate has been reported to be in the range of 4-20\%. It seems to be equal in men and women, though peaking in the fourth decade. It is stated to account for $5 \%$ of deaths in young adults and $25 \%$ deaths in athletes. ${ }^{2}$

Patients with ARVC may have a wide range of presenting symptoms, varying from fatigue, atypical chest pain, syncope or acute coronary syndrome. The main presenting features include arrhythmias, sudden cardiac death or even heart failure. Approximately $90 \%$ of patients present with ECG abnormalities. The important ones include $\mathrm{T}$ wave inversions in leads $\mathrm{V} 1-\mathrm{V} 3$, which are seen in about $54 \%$ of the patients (in the absence of RBBB). ${ }^{3}$ Epsilon waves, which are post excitation electrical potentials that occur at the end of QRS complex are seen in $30 \%$ of all cases of ARVC. They are very specific of ARVC and are due to delayed RV activation. ${ }^{4}$

Major echocardiographic findings include RV dilation, localized aneurysms, enlarged left atria (LA), dilated RV out flow tract, increased reflectivity of moderator band, prominent trabeculations of $\mathrm{RV}$ apex and inferobasal dyskinesis. MRI is considered to be the one of the important and accurate non-invasive tests available to diagnose ARVC. It helps to analyse RV anatomically and functionally. It also identifies intramyocardial fat deposits, wall thinning, hypertrophy, RVOT enlargement and RV dilatation and aneurysm .

$\beta$-blockers and amiodarone are considered useful for patients with asymptomatic ARVC to suppress adrenergically stimulated arrhythmias. Intracardiac defibrillatiors (ICD) should be implanted in for the prevention of SCD in patients with ARVD/ARVC include documented sustained ventricular tachycardia or fibrillation for those who are receiving optimal medical therapy and have a reasonable expectation of survival (Class I recommendation). For primary prevention of ICD implantation can be considered (Class II a recommendation) in patients with extensive disease, including those with LV involvement, with one affected family member with SCD, or undiagnosed syncope when Ventricular Tachycardia (VT) or Ventricular Fibrillation (VF) has not been excluded as the cause of syncope, and who are receiving optimal medical therapy and have a reasonable expectation of survival. ${ }^{5}$

\section{Conclusion}

ARVC is an important cause of sudden cardiac death in young individuals. It is an in herited condition characterized by fibrofatty replacement of the right ventricle. Diagnosis should always be suspected in young patients who have history of resuscitated cardiac arrest or aborted sudden cardiac death, syncope and ventricular arrhythmias in the past. The clinical presentation can be varied from asymptomatic to life threatening arrhythmias. This case highlights the role of basic $2 \mathrm{D}$ echocardiography to identify suspected ARVC case along with multimodality imaging with cardiac MRI to make a definite diagnosis of ARVC.

\section{References}

1. Frank R, Fontain G. Electrocardiologie de quatrecas de dysplasia ventriculair edroite arythmogene. Arch Mal Coeur Vaiss 1978;71:963-72.

2. Kies P, Bootsma M, Bax J, et al. Arrhythmogenic right ventricular dysplasia/cardiomyopathy: screening, diagnosis, and treatment. Heart Rhythm 2006;3:225-34. https://doi.org/10.1016/j.hrthm.2005.10.018

3. Fontaine G, Fontaliram F, Herbert JL, et al. Arrhythmogenic right ventricular dysplasia. Ann Rev Med 1999;50:17-35 https://doi.org/10.1146/annurev.med.50.1.17

4. Gemayel C, Pelliccia A, Thompson PD. Arrhythmogenic right ventricular cardiomyopathy. J Am Coll Cardiol 2001;38:177381. https://doi.org/10.1016/S0735-1097(01)01654-0

5. Pelliccia A, Corrado D, Bjornstad HH, et al. Recommendations for participation in competitive sport and leisure-time physical activity in individuals with cardiomyopathies, myocarditis and pericarditis. Eur J Cardiovasc Prev Rehabil 2006;13:87685. https://doi.org/10.1097/01.hjr.0000238393.96975.32 